\title{
The inheritance of style length in Oxalis rosea
}

J. H. Bennett, Carolyn R. Leach and

I. R. Goodwins
Department of Genetics, University of Adelaide, Adelaide, South Australia, 5001.

In Oxalis rosea it is found that some short-styled plants are dominant to non-short (mid- or long-styled plants) whilst others are recessive. Breeding experiments show that the short style form is under the control of two gene pairs ( $A$, a and $S, s$ ) with shorts having either of the genotypes aaSS and aaSs. In plants segregating for $A, a$ on an $S S$ background, shorts appear as recessive whilst in plants segregating for $S, s$ on an aa background, shorts appear as dominant. Among non-shorts, mids $(M M, M m)$ are dominant to long $(\mathrm{mm})$. Genes at the three loci (i) $A, a,(i i) S, s$ and (iii) $M, m$ show independent assortment. These results are compared with previous suggestions of recessive short in $O$. rosea by von Ubisch (1926) and in $O$. articulata by Fyfe (1956).

\section{INTRODUCTION}

Fyfe (1956) first drew attention to the existence of two different genetic systems controlling style length inheritance in different tristylic species of Oxalis. In some species, the short-style form was found to be dominant to non-short (mid- or longstyle forms) whilst in other species short was recessive to non-short. Fyfe named four Oxalis species having a dominant short $(O$. valdiviensis, $O$. hirta, O. tragapoda, O. boweii) and two other species in which short was said to be recessive ( $O$. articulata and $O$. rosea). She presented her own genetical data for $O$. articulata indicating that short is recessive whilst for $O$. rosea she referred to the work of von Ubisch (1926). Subsequently Mulcahy (1964) reported that Fyfe had stated in a personal communication that when she sent some of her specimens of $O$. articulata to von Ubisch, the latter had replied that the material was taxonomically identical to what she, von Ubisch, had identified as $O$. rosea. Mulcahy says that "the actual identity of the specimens variously referred to as $O$. articulata or $O$. rosea was not indicated by Fyfe" and he also comments that, "since it is extremely unlikely that a single species could possess two genetic systems, it is apparent that at least one and perhaps even both of the proposed systems may be incorrect". What Mulcahy here describes as "two genetic systems" are Fyfe's two- locus model and von Ubisch's three-locus model, which we shall discuss later. The present study was undertaken in the hope of removing some of the confusion as to the mode of inheritance of the short style form in $O$. rosea.

\section{MATERIALS AND METHODS}

Seed of $O$. rosea was obtained from the Royal Botanic Gardens, Kew, England. Following seed germination, plants of the three style forms were established and maintained in pots in a glasshouse. The plants of this species are clearly distinguishable from those of $O$. articulata cultivated by Fyfe. Plants of all style forms in $O$. rosea may readily be induced to set seed either by self-pollination or by intercrossing. Crosses of both kinds were used in this study.

\section{RESULTS}

The foundation plants comprised four shorts, seven mids and one long. Crosses made between these shorts and mids gave the progenies shown in table 1. Whilst some of these progenies contain approximately half shorts and half mids, others show an apparent deficiency of shorts. 
Table 1 Results of crosses of foundation short- and mid-styled plants

\begin{tabular}{lcccc}
\hline & \multicolumn{3}{c}{ Style forms of progeny } & \\
\cline { 2 - 4 } Cross & Short & Mid & Long & Total \\
\hline S1 $\times$ M1* & 17 & 32 & - & 49 \\
S1 $\times$ M2 & 28 & 40 & - & 68 \\
S2 $\times$ M2 & 26 & 43 & - & 69 \\
S2 $\times$ M3 & 11 & 10 & - & 21 \\
S1 $\times$ M4 & 6 & 7 & - & 13 \\
S3 $\times$ M2 & 30 & 30 & - & 60 \\
S3 $\times$ M1 & 1 & 6 & - & 7 \\
\hline
\end{tabular}

* $\mathrm{S}$ and $\mathrm{M}$ refer to short- and mid-style forms respectively

In addition to the crosses between the foundation shorts and mids shown in table 1 , a cross was made between two foundation mids (M3 and M5) and gave three short and six mid progeny whilst seed obtained from one of the foundation mids (M4), presumably after self-pollination, led to seven plants, all mids. The occurrence of shorts in the progeny of a cross between two mids suggests that short is recessive to non-short. Further support for this suggestion was obtained from selfed progenies raised from four of the seven mids derived from M4 (see table 2). The first and second progenies in table 2 presumably came from plants that were homozygous mid. The presence of shorts in the third and fourth progenies is in agreement with the suggestion that short is recessive to nonshort, although the proportion of shorts is less than 25 per cent.

Table 2 Progenies of four mid plants following self-pollination

\begin{tabular}{lllll}
\hline & \multicolumn{3}{c}{ Style forms of progeny } & \\
\cline { 2 - 4 } Mid parent & Short & Mid & Long & Total \\
\hline (i) & - & 16 & - & 16 \\
(ii) & - & 35 & - & 35 \\
(iii) & 4 & 30 & - & 34 \\
(iv) & 3 & 33 & - & 36 \\
\hline
\end{tabular}

If short is recessive to non-short, then a nonshort plant having a short parent will be expected on self-pollination to give some short progeny. One of the 32 mid progeny shown in the top row of table 1 was self-pollinated and a progeny of 37 plants was scored. This progeny included 24 mids and 13 longs. The absence of short from this progeny suggests that short cannot be a simple recessive. It also seems that mid is dominant to long, the parental mid presumably being heterozygous $\mathrm{Mm}$.

One of the 13 longs $\left(\mathrm{L}^{*}\right)$ was crossed with one of the short progeny $\left(\mathrm{S}^{*}\right)$ from mid parent (iv) in table 2 and gave 180 progeny, all shorts. This clearly indicates that short can behave as dominant to non-short and further that $\mathrm{S}^{*}$ was homozygous for dominant short. But how can we explain the occurrence of such a plant $\mathrm{S}^{*}$ in the progeny of a self-pollinated mid-styled plant? And how is it possible to reconcile the separate but clear indications of recessive and dominant shorts in the one species?

It seems that the simplest model which can account for these observations is one with two loci controlling the short vs non-short difference with a gene $S$ (say) resulting in short style only in plants that are homozygous $a a$. The genotypes of shorts would then be either aaSS or aaSs whilst plants with other genotypes at these two loci would be non-short. In plants segregating for $A$ and $a$ on an $S S$ background, short would appear as recessive whilst in plants segregating for $S$ and $s$ on an $a a$ background, short would appear as dominant. Non-shorts with the genotype $A a S s$ would lead on selfing or intercrossing to progeny with an expected segregation ratio of 3 short: 13 non-short if there were independent assortment of genes at the two loci. The observed segregations in the selfed progenies of mid parents (iii) and (iv) shown in table 2 are in agreement with this ratio $\left(\chi^{2}=3 \cdot 52\right.$ with $1 \mathrm{df}$ ).

The mid parent of the long $\mathrm{L}^{*}$ had a short parent and so cannot be homozygous $A A$. If we note the absence of shorts and interpret the observed segregation of 24 mids: 13 longs in the progeny of this selfed mid as a $3: 1$ ratio with mids $(M M, M m)$ dominant to long $(\mathrm{mm})$, we may conclude that the genotype of this mid is either Aass $\mathrm{Mm}$ or aass $\mathrm{Mm}$. The cross $\mathrm{S}^{*} \times \mathrm{L}^{*}$ was then presumably of the following kind aaSSMM $\times$ aassmm with all progeny being shorts and having the genotype aaSsM. Six of the 180 short progeny were self-pollinated with the results shown in table 3 . The observed segregation of 86 short, $13 \mathrm{mid}$ and 6 long is in good agreement with the frequencies 12 short: 3 mid: 1 long which would be expected with independent assortment $\left(\chi^{2}=2.99\right.$ with $2 \mathrm{df}$ ). There is no suggestion here of close linkage between $S$ and $M$ such as Fisher and Martin (1948) found with $O$. valdiviensis. Indeed, if $S$ and $M$ were linked, then since the self-pollinated shorts must be in coupling we could estimate the recombination frequency as $0.44 \pm 0.09$. 
Table 3 Progenies of six short plants following self-pollination

\begin{tabular}{lcccc}
\hline & \multicolumn{3}{c}{ Style forms of progeny } & \\
\cline { 2 - 4 } Short parent & Short & Mid & Long & Total \\
\hline (i) & 17 & 2 & 3 & 22 \\
(ii) & 24 & 4 & - & 28 \\
(iii) & 13 & 4 & 1 & 18 \\
(iv) & 20 & 1 & - & 21 \\
(v) & 8 & 2 & 2 & 12 \\
(vi) & 4 & - & - & 4 \\
\hline Total & 86 & 13 & 6 & 105 \\
\hline
\end{tabular}

\section{DISCUSSION}

The three-locus model here proposed for the genetical control of tristyly in $O$. rosea is shown in table 4 . There are $3^{3}=27$ genotypes, 6 for shorts, 14 for mids and 7 for longs. Fisher and Martin's (1948) model for the genetical control of tristyly in $O$. valdiviensis may be seen as a degenerate case of the three-locus model with the $\boldsymbol{A}$ gene absent (see table 5). Similarly, Fyfe's model for the control

Table 4 Genotypes for short-, mid- and long-styled plants according to the three-locus model for genetical control of style length in Oxalis rosea

\begin{tabular}{lll}
\hline Short & Mid & Long \\
\hline aaSSMM & AASSMM & AASSmm \\
aaSSMm & AASSMm & AASsmm \\
aaSSmm & AASsMM & AAssmm \\
aaSsMM & AASsMm & AaSSmm \\
aaSsMm & AAssMM & AaSsmm \\
aaSsmm & AAssMm & Aassmm \\
& AsSSMM & aassmm \\
& AaSSMm & \\
& AsSsMM & \\
& AaSsMm & \\
& AassMM & \\
& Aass Mm & \\
& aass MM & \\
& aass Mm & \\
& & \\
\end{tabular}

Table 5 Genotypes for short-, mid- and long-styled plants according to Fisher and Martin's (1948) model for genetical control of tristyly in Oxalis valdiviensis

\begin{tabular}{lll}
\hline Short & Mid & Long \\
\hline aaSSMM & $\begin{array}{l}\text { aassMM } \\
\text { aassMm }\end{array}$ & aassmm \\
aaSSmm & & \\
aaSsMM & & \\
aaSsMm & & \\
aaSsmm & & \\
\hline
\end{tabular}

of tristyly in $O$. articulata may be seen as a degenerate case with the $s$ gene absent (see table 6).

Table 6 Genotypes for short-, mid- and long-styled plants according to Fyfe's (1956) model for genetical control of tristyly in Oxalis articulata

\begin{tabular}{lll}
\hline Short & Mid & Long \\
\hline aaSSMM & AASSMM & AASSmm \\
aaSSMm & AASSMm & AaSSmm \\
aaSSmm & AaSSMM & \\
& AaSSMm & \\
\hline
\end{tabular}

Fyfe's suggestion that short is recessive to nonshort in $O$. articulata stemmed from her finding that on crossing a long and a mid, where both plants had one short parent, shorts reappeared in the progeny. Her total counts for the progeny of these crosses were 47 long, 107 mid and 76 short. The proportion of shorts is more than 25 per cent, but, as Fyfe noted, the performance of crosses in the following year removed any doubt that some unknown factor had influenced that ratio. When ten of the 47 longs were crossed to a short, three gave only longs and seven gave 50 per cent longs and 50 per cent shorts. When five of the 107 mids were selfed, one gave only mids and longs and four gave 25 per cent shorts. Fyfe does not discuss the $107 \mathrm{mid}: 47$ long ratio observed in the progeny of her long $\times$ mid cross. If there were independent assortment of genes at the two loci, we would expect 50 per cent of the non-short progeny to be longs but a ratio of approximately 2 mid: 1 long could be obtained with close linkage. Whilst there may be a case for further study of the role of linkage in style length inheritance in $O$. articulata, Fyfe's data seem amply to support her suggestion that short is recessive and epistatic to mid in this species. It therefore seems unfortunate that Mulcahy (1964) has published, without any explanation, a "reinterpretation" of Fyfe's material with a quite different genetic model for tristyly in $O$. articulata. In Mulcahy's account, short is neither recessive to non-short nor epistatic to mid, two features that are an essential part of Fyfe's model. von Ubisch (1926) put forward a complicated three-locus model to account for her early genetical data on tristyly, supposedly in $O$. rosea (although, as indicated before, the species she worked with was apparently $O$. articulata). This model is shown in table 7 using the same symbolism as in table 4 . (To convert to von Ubisch's symbolism, replace $a, A$ by $C, c, S, s$ by $D, d$ and $M, m$ by $B, b$ respectively.) Short is shown as a simple recessive and 
Table 7 Genotypes for short, mid- and long-styled plants according to von Ubisch's (1926) model for genetical control of tristyly in Oxalis rosea

\begin{tabular}{lll}
\hline Short & Mid & Long \\
\hline aaSSMM & AASSMM & AASSmm \\
aaSSMm & AASsMM & AASsmm \\
aaSSmm & AASsMm & AAssmm \\
aaSsMM & AsSSMM & AaSSmm \\
aaSsMm & AaSsMM & AaSsmm \\
aaSsmm & AaSsMm & Aassmm \\
aassMM A $^{*}$ & AAssMM & AASSMm* \\
aass Mm & AAssMm & AaSSMm* \\
aassmm & AassMM & \\
& AassMm & \\
\hline
\end{tabular}

With the model that we have presented (see table 4), the four genotypes marked * correspond with the mid phenotype whilst the genotype marked + corresponds with the long phenotype.

epistatic to mid, the homozygous geno:ype $a a$ being short irrespective of the genotypes at the other two loci. von Ubisch's assignment of genotypes to the mid and long phenotypes is rather curious. Mids are said to have the genotype $A A$ or $A a$ with any of $S S M M, S s M M, S s M m$, ssMM or $s s M m$ whilst longs are $A A$ or $A a$ with any of $S S M m, S S m m, S s m m$ or $s s m m$. von Ubisch said $S$ was rarely detected and occurred only if $M$ were present. We are unable to see any rational genetic explanation of genotype-phenotype correspon- dences for the mid $v$. long difference of the kind involved in von Ubisch's model. She suggests that genotypes $M M$ and $M m$ give the mid phenotype except when $M m$ occurs with $S S$ when the phenotype is long. von Ubisch did not report any critical crosses being made to test this suggestion. The model that we have proposed for $O$. rosea (see table 4) seems to provide a satisfactory explanation for her data. von Ubisch's model does not seem to merit further genetical consideration, although it has, from time to time, become the subject of serious mathematical study (Finney, 1952; 1983; Moran, 1962).

\section{REFERENCES}

FINNEY, D. J. 1952. The equilibrium of a self-incompatible polymorphic species. Genetica, 26, 33-64.

FINNEY, D. J. 1983. Genetic equilibria of species with selfincompatible polymorphisms. Biometrics, $39,573-585$.

FISHER, R. A. AND MARTIN, V. C. 1948. Genetics of style length in Oxalis. Nature, 162, 533.

FYFE, V. C. 1956. Two modes of inheritance of the short-styled form in the genus Oxalis. Nature, 177, 942-943.

MORAN, P. A. P. 1962. The Statistical Processes of Evolutionary Theory. Clarendon Press, Oxford. pp. 159.

MUlCAHY, D. L. 1964. The reproductive biology of Oxalis priceae. Amer. Jour. Bot., 51, 1045-1050.

UBISCH, G. VON 1926. Koppelung von Farbe und Heterostylie bei Oxalis rosea. Biol. Zentralblatt., 46, 633-645. 